\title{
Origins of ambiguity in the inversion of remote sensing reflectance signals by spectral matching in optically complex shelf seas
}

Danielle Creanor

danielle.creanor@strath.ac.uk

\section{Alex Cunningham}

a.cunningham@strath.ac.uk
Physics Department, University of Strathclyde, 107 Rottenrow, Glasgow G40NG, UK

Physics Department, University of Strathclyde, 107 Rottenrow, Glasgow G40NG, UK

The reported occurrence of multiple solutions in the inversion of remote sensing reflectance, $R_{r s}$, signals is of considerable significance for attempts to recover the concentrations of optically significant materials (OSMs) in shelf seas. The severity of this problem was assessed by quantifying the number of matches between individual multi wavelength remote sensing "observations" and the entries in a pre-computed look-up table (LUT) spanning the range of possible observations. As a simplifying step, radiative transfer modeling was used to confirm the existence of a linear relationship between $R_{r s}$ in the visible wavebands and the ratio of the backscattering to absorption coefficients, $b_{b} / a$ over the full range of OSM concentrations likely to be found in shelf seas. This meant that an LUT of appropriate size and resolution could be constructed from $b_{b} / a$ vectors rather than $R_{r s}$ spectra, with a considerable saving of computational effort. The number of matches in the LUT was then determined as a function of the degree of noise present in the observation and the strictness of the matching criterion (a simple least-squares fitting routine). Perfect matching for the six visible wavebands of the SeaWiFs satellite radiometer was achieved only when the "observed" $b_{b} / a$ vector was represented exactly in the library. The introduction of noise representing observational errors rapidly led to multiple matches, as did relaxation of the matching criterion. As an example of the sensitivity of OSM recovery to errors of observation, it was found that an average error of $0.1 \%$, statistically distributed across all wavebands, led to an average recovery error of $6.4 \%$ in CHL for 3000 randomly selected observations. [DOI: 10.2971/jeos.2010.10018s]

Keywords: shelf sea optics, inherent optical properties, spectral matching, SeaWiFs

\section{INTRODUCTION}

Optical remote sensing using satellite-borne radiometers has become an important tool for studying biological and physical processes in ocean basins [1, 2], and is recognized as being potentially of great value for monitoring the changing status of coastal waters and shelf seas [3]. Unfortunately, remote sensing algorithms derived using global data sets are not reliable when applied to waters subject to terrestrial influence [4]. The problem was foreseen over 30 years ago by Morel and Prieur [5], who drew a distinction between waters where optical variability is correlated with phytoplankton population density and those where this correlation is disrupted by the presence of a wider range of optically significant materials (OSMs). Shelf seas generally fall into the latter category. In the simplest analysis, OSMs fall into three classes: phytoplankton cells (measured as chlorophyll concentration, CHL), suspended mineral particles (measured as the dry weight of mineral suspended solids per unit filtered volume, $M S S$ ) and dissolved coloured organic material (measured as the absorption coefficient of filtered samples at $440 \mathrm{~nm}, C D O M)$. The problem of inversion in marine remote sensing consists of deriving OSM concentrations from measurements of remote sensing reflectance, $R_{r s}$, in the visible waveband. It appears at first sight to be a reasonably tractable one, since $R_{r s}$ values under typical satellite imaging conditions (high viewing angles and clear skies) are mainly determined by the inherent optical properties (IOPs) of the water column which are linear functions of OSM concentrations over the range of interest. A minimal set of determining IOPs consists of the coefficients of absorption $a$, and scattering $b$ and the volume scattering function $\beta$, all of which are functions of wavelength, $\lambda$. In numerical models, $\beta$ is often approximated by a generic scattering function, parameterised to reproduce the required scattering to backscattering ratio, $b_{b} / b[6,7]$.

Water column IOPs can be expressed as the sum of the products of the concentrations of the OSMs and their specific inherent optical properties (SIOPs), which are generally designated in the form $a^{*}, b^{*}$, etc. For example, the total absorption coefficient $a$ is given by

$$
a=a_{w}+\left(a_{c h l}^{*} \times C H L\right)+\left(a_{m s s}^{*} \times M S S\right)+\left(a_{c d o m}^{*} \times C D O M\right)
$$

where $w$ refers to pure water, and the other terms are as previously defined. It has long been known that the irradiance reflectance just below the sea surface, $R$, is a single valued function of the coefficients of backscattering, $b_{b}$, and absorption, $a[6,7]$. For remote sensing reflectance, $R_{r s}$, defined as the ratio of the water leaving radiance to the downward irradiance just above the sea surface

$$
\begin{aligned}
& R_{r s}=\frac{\left(1-r_{F}\right) f_{L, \beta}}{n^{2} Q} \times \frac{b_{b}}{a}=G \\
& \times \frac{b_{b, w}+\left(b_{b, c h l}^{*} \times C H L\right)+\left(b_{b, m s s}^{*} \times M S S\right)+\left(b_{b, c d o m}^{*} \times C D O M\right)}{a_{w}+\left(a_{c h l}^{*} \times C H L\right)+\left(a_{m s s}^{*} \times M S S\right)+\left(a_{c d o m}^{*} \times C D O M\right)} .
\end{aligned}
$$


The factor $f_{L, \beta}$ in Eq. (2) depends on the radiance distribution $L$ and the volume scattering function of the water $\beta, Q$ is the ratio of upwelling irradiance to radiance, $r_{F}$ is the Fresnel reflectance at the air/water interface and $n$ is the refractive index of seawater [8]. For conditions where $L$ and $\beta$ are held constant, $G$ is the gradient of the linear relationship between $R_{r s}$ and $b_{b} / a$. One important consequence of the occurrence of a ratio on the right hand side of Eq. (2) is that spectral unmixing techniques, which are successfully used in terrestrial remote sensing [9], cannot be applied to marine water columns because remote sensing reflectances do not respond linearly to changes in the concentrations of the non-water constituents. A range of approaches to the remote sensing inversion problem have been formulated in the form of neural networks [10], site specific algorithms [11] and "semi-analytical" algorithms [12, 13]. However, for Case 2 waters, no robust and generally applicable solutions have been found. One promising method which has not been fully explored is spectrum matching, in which an observed spectrum is matched to a set of possible OSM concentrations by querying a comprehensive look-up table $[14,15]$. This immediately raises the question of whether the inversion problem is well conditioned: that is, is there a unique relationship between particular combinations of material concentrations and the resulting remote sensing reflectances? Inspection of Eq. (2) indicates that this is not the case if only a single reflectance waveband is considered. The question was addressed for simultaneous observations in multiple wavebands in a pioneering paper by Defoin-Patel and Chami [16], who concluded that solutions of the inverse problem in optically complex shelf seas were frequently multivalued. However, the methodology used by these authors was based on statistical sampling of an extensive field data set [17], and did not identify whether the ambiguity arose from intrinsic multiple solutions, real variability in the specific IOPs, or possible errors of observation. Consequently, the present paper explicitly examines the potential origins of ambiguity in solutions to the $R_{r s}$ inversion problem in optically complex seas.

\section{METHODS}

The obvious way to tackle the problem would be to create a look-up table (LUT) of $R_{r s}$ spectra from radiative transfer calculations covering the range of OSM concentrations likely to be found in shelf seas, and then search it to find matches to 'observed' spectra. However, the quality of the matches achieved depends on the spacing of the LUT entries, and the construction of a table with sufficient resolution to be useful in practice would require a very large number of radiative transfer calculations. A solution to this problem can be found in the relationship between $b_{b} / a$ and $R_{r s}$ displayed in Eq. (2): if the constant $G$ is known as a function of wavelength, then observed $R_{r s}$ values are uniquely mapped on to $b_{b} / a$ values. It is then only necessary to generate an LUT of $b_{b} / a$ vectors in wavebands matching those of the $R_{r s}$ observations, and this is easily calculated from the products of the OSM concentrations and SIOPs.

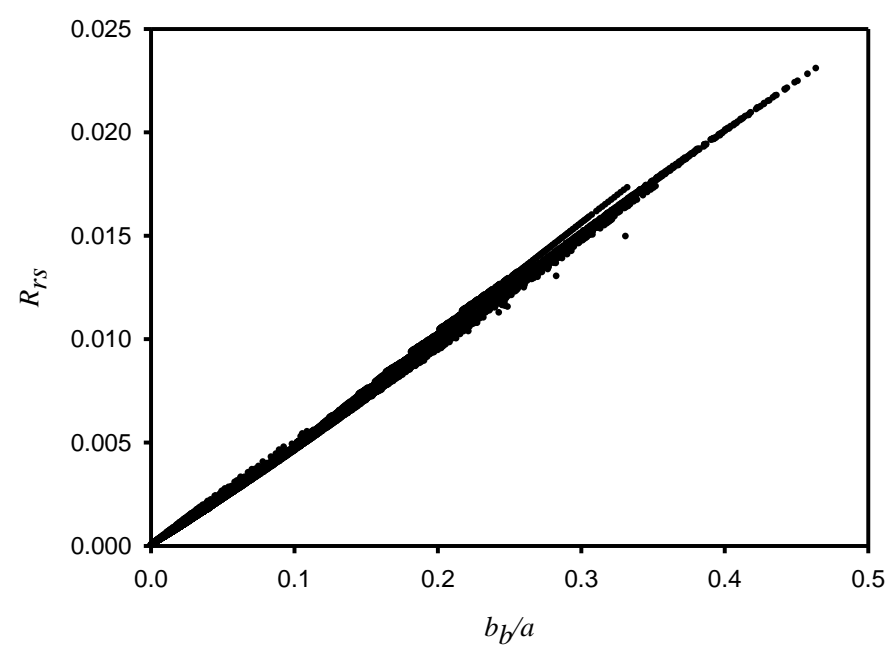

FIC. 1 Hydrolight radiative transfer calculations of the relationship between remote sensing reflectance, $R_{r s}$, and the backscattering to absorption ratio, $b_{b} / a$, for all wavebands listed in Table 1 and OSM concentrations listed in Section 2.1.

\subsection{Validation of Eq. (2)}

SIOPs in the seven visible wavebands used by the SeaWiFs satellite radiometer were derived from in situ measurements of optical properties and seawater composition in the Irish and Celtic Seas, using the methodology documented in [18]. It was necessary to interpolatate between measurements to match the wavebands used in the model, and the results are listed in Table 1. These values were incorporated in radiative transfer calculations using the Hydrolight software package [7] in order to validate the applicability of Eq. (2), and incidentally determine appropriate values for $G$ in SeaWiFs wavebands. The calculations were carried out for a solar zenith angle of $45^{\circ}$, an infinitely deep water column, wind speed of 3.1 $\mathrm{ms}^{-1}$ and zero cloud cover. They covered the range of constituent concentrations and combinations that might normally be found in a tidally stirred shelf sea, with $C H L$ ranging from $0 \mathrm{mg} / \mathrm{m}^{3}$ to $20 \mathrm{mg} / \mathrm{m}^{3}$, MSS from $0 \mathrm{~g} / \mathrm{m}^{3}$ to $20 \mathrm{~g} / \mathrm{m}^{3}$ and CDOM (as a440) from $0 \mathrm{~m}^{-1}$ to $1 \mathrm{~m}^{-1}$. For each constituent, the range was divided into 10 equal increments. All possible combinations including null values were examined, giving a total of 1331 radiative transfer calculations.

The results, which are illustrated in Figure 1, confirmed the linear relationship between $b_{b} / a$ and $R_{r s}$ in Eq. (2) for all wavebands and the full range of OSM concentrations. The gradient of the lines varied slightly with wavelength due to spectral variation in the $b_{b} / b$ ratio used to generate the scattering phase function. Linear least-squares fits to the data gave $G$ values of 0.046 at $412 \mathrm{~nm} ; 0.048$ at $443 \mathrm{~nm} ; 0.049$ at $489 \mathrm{~nm}$; 0.050 at $510 \mathrm{~nm} ; 0.051$ at $555 \mathrm{~nm} ; 0.052$ at $665 \mathrm{~nm}$ and 0.052 at $700 \mathrm{~nm}$. The associated coefficients of determination were above 0.99 in all cases.

\subsection{Construction of a look-up table of $b_{b} / a$ vectors}

The LUT was calculated using expressions of the form shown in Eq. (1) to generate total $b_{b}$ and $a$ values from the SIOPs listed in Table 1. In this case OSM concentration ranges were restricted to $0 \mathrm{mg} / \mathrm{m}^{3}$ to $10 \mathrm{mg} / \mathrm{m}^{3}$ for $C H L, 0 \mathrm{~g} / \mathrm{m}^{3}$ to 


\begin{tabular}{|c|c|c|c|c|c|c|c|c|c|c|}
\hline Band & \multicolumn{3}{|c|}{ Water } & \multicolumn{3}{c|}{ CHL } & \multicolumn{3}{|c|}{ MSS } & CDOM \\
\hline$(\mathrm{nm})$ & $a^{*}$ & $b_{b}^{*}$ & $b^{*}$ & $a^{*}$ & $b_{b}^{*}$ & $b^{*}$ & $a^{*}$ & $b_{b}^{*}$ & $b^{*}$ & $a^{*}$ \\
\hline 413 & 0.005 & 0.0035 & 0.0067 & 0.036 & 0.0012 & 0.0509 & 0.071 & 0.0154 & 0.3134 & 1.556 \\
\hline 443 & 0.007 & 0.0026 & 0.0048 & 0.038 & 0.0012 & 0.0395 & 0.057 & 0.0145 & 0.3226 & 0.969 \\
\hline 489 & 0.015 & 0.0017 & 0.0031 & 0.025 & 0.0012 & 0.0464 & 0.041 & 0.0133 & 0.3305 & 0.503 \\
\hline 510 & 0.033 & 0.0016 & 0.0026 & 0.020 & 0.0011 & 0.0482 & 0.035 & 0.0127 & 0.3340 & 0.386 \\
\hline 555 & 0.059 & 0.0012 & 0.0019 & 0.011 & 0.0011 & 0.0544 & 0.022 & 0.0115 & 0.3340 & 0.226 \\
\hline 665 & 0.425 & 0.0005 & 0.0008 & 0.024 & 0.0011 & 0.0435 & 0.005 & 0.0086 & 0.3435 & 0.036 \\
\hline 700 & 0.624 & 0.0003 & 0.0007 & 0.013 & 0.0011 & 0.0516 & 0.001 & 0.0077 & 0.3428 & 0.009 \\
\hline
\end{tabular}

TABLE 1 Specific inherent optical properties for pure seawater and the three optically significant materials at SeaWiFs wavebands.

$10 \mathrm{~g} / \mathrm{m}^{3}$ for $M S S$ and $0 \mathrm{~m}^{-1}$ to $1 \mathrm{~m}^{-1}$ for CDOM. Entries were spaced at numerical intervals of 0.1 for $C H L$ and MSS and 0.01 for CDOM. The resulting table of $b_{b} / a$ vectors for all possible combinations of the three OSM concentrations had 1,030,301 entries.

\subsection{Spectrum matching}

The matching of six-waveband "observed" vectors with entries in the LUT was based on the calculation of the root mean square error (RMSE) averaged over all wavebands. This measure of spectral distance differs from the one used by DefoinPlatel and Chami [16], who calculated the maximum fractional difference waveband by waveband. For a single observed vector, the calculation of all RMSE values took a few seconds using MATLAB on a desktop computer: the time required could be significantly reduced by optimising the software used. There are, however, two obvious problems associated with the practical application of LUTs. First, errors of measurement might corrupt the observed vector and lead to spurious matches. Second, since any LUT has finite resolution, the observed vector might occupy a gap between entries in the table. Errors of measurement were simulated in this study by adding Gaussian noise independently to each waveband with a mean equal to the "true" value and a standard deviation that was varied as a test of sensitivity. The effect of limited LUT resolution was investigated by relaxing the matching criteria to accept all library entries within a cluster defined by a variable $R M S E$ rather than simply choosing the RMSE closest to zero.

\section{RESULTS}

\subsection{The effect of measurement errors and acceptance criteria on the number of matches returned}

One hundred $b_{b} / a$ vectors were selected at random from the LUT and perturbed by a varying degree of noise applied independently to each waveband. The noise level was selected from a Gaussian distribution whose mean was equal to the initial $b_{b} / a$ value and whose standard deviation was set at a variable percentage $(0 \%$ to $5 \%)$ of the mean. $5 \%$ corresponds to the target uncertainty for the recovery of water-leaving radiances from SeaWiFs and MODIS observations, but this figure is exceeded by at least a factor of two in practice [19]. The quality of the matches in the LUT was assessed by calculating the RMSE for the $b_{b} / a$ vector averaged over all wavebands.

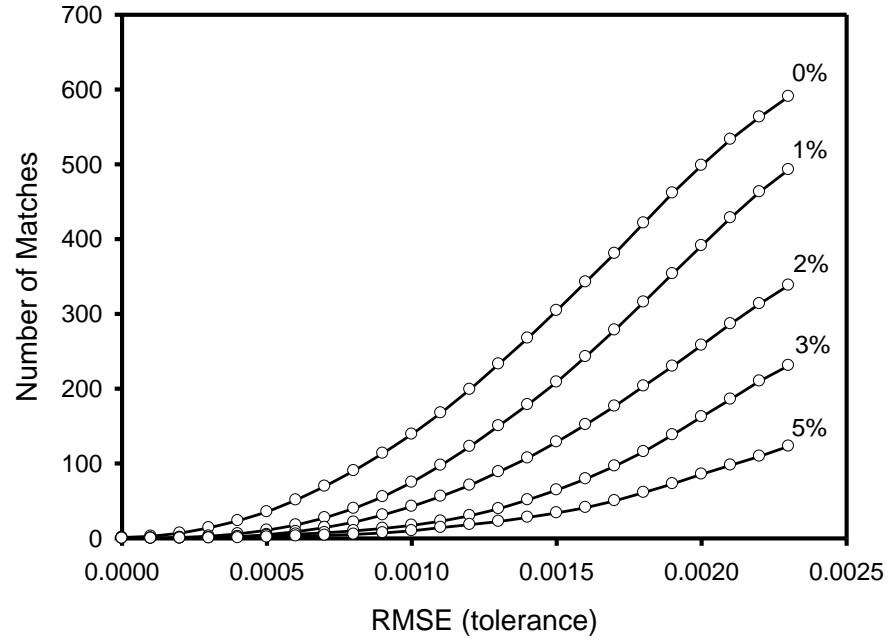

FIG. 2 Number of matches returned within a given acceptance interval (RMSE) for $b_{b} / a$ vectors to which Gaussian noise had been added in the range $0 \%$ to $5 \%$.

Matches were generated with acceptance criteria increasing from 0 to 0.01 RMSE in steps of 0.0001 . Figure 2 shows the combined effect of noise and acceptance tolerance on the number of $b_{b} / a$ vectors recovered by the matching process as an average for the 100 spectra used in the test. If an exact replica of the observed spectrum was found in the library, then only one match was returned with the minimum RMSE. This was an important result, which (i) indicated that no intrinsic ambiguity was found in the six-waveband matching process, and (ii) confirmed that there was a unique relationship between $b_{b} / a$ vectors and the OSM concentration triplets used to generate the LUT. The number of potential matches increased rapidly as the acceptance tolerance was increased (an RMSE of 0.0024 produced 618 matches for noise-free spectra). The addition of random noise to the "observed" spectrum reduced the number of matches produced for a given acceptance interval because a higher proportion of the modified spectra fell outside the acceptance criteria. Quantifying the number of $b_{b} / a$ matches did not, however, give any indication of the quality of OSM concentration recoveries that could be achieved and this is explored further below.

\subsection{The effect of errors of observation on the recovery of OSM concentrations}

For brevity, the recovery of $C H L$ concentrations is used as an example in this section; other OSMs followed a very similar pattern. A set of $100 b_{b} / a$ vectors was selected at random from 


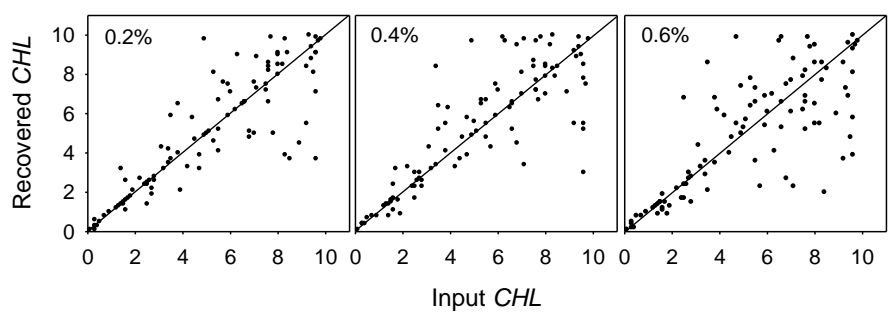

FIG. 3 Degradation in CHL recovery as noise is added to the observed $b_{b} / a$ vector with standard deviations (from left to right) of $0.2 \%, 0.4 \%$ and $0.6 \%$ of the mean value. For each recovery, the LUT match with the lowest RMSE was selected.

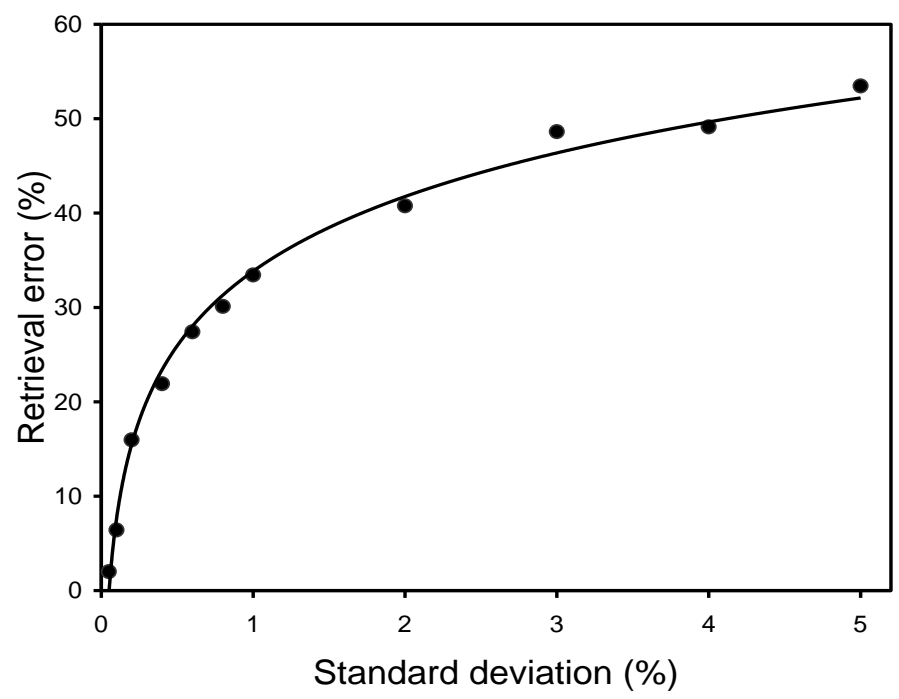

FIG. 4 Variation in the average percentage error in CHL recovery for 3000 randomly selected spectra as the standard deviation of the added noise increases.

the LUT and noise added to each waveband as described in Section 3.1. The LUT was then searched and the match with the lowest RMSE for each vector accepted. Given the unique mapping of OSM concentration triplets on $b_{b} / a$ vectors, the recovery of OSM concentrations became a trivial operation. Figure 3 illustrates the degradation in accuracy of $\mathrm{CHL}$ recovery in response to added noise for 100 randomly selected $b_{b} / a$ vectors. Perfect recoveries were achieved when zero noise was added. The average RMSE values for the 100 "observations" provide a useful measure of the quality of the matches achieved. Values were $0.00026,0.00043$ and 0.00063 for standard deviations of $0.2 \%, 0.4 \%$ and $0.6 \%$, respectively.

In order to gain a clearer picture of the significance of errors of observation for the inversion process, a total of 3000 entries in the LUT were chosen at random, degraded by the addition of noise as previously described, and then subjected to the leastsquares matching procedure. Figure 4 shows how the average percentage error in $\mathrm{CHL}$ recovery increases with the standard deviation of the added noise, expressed as a percentage of the undegraded value; the line drawn through the points is of the form $y=11.4 \ln (x)+34$.

\section{DISCUSSION AND CONCLUSIONS}

Two main approaches are employed for the retrieval of OSM concentrations from $R_{r s}$ spectra. One derives relationships be- tween $R_{r s}$ features (such as band ratios) and OSM values by applying statistical regression techniques to large data sets. The other, which is investigated here, is based on matching $R_{r s}$ spectra to entries in a reference library created using a predictive model. This paper demonstrates that the use of an LUT based on $b_{b} / a$ vectors offers an efficient means of assessing the performance of the spectral matching approach. There was no ambiguity in matching retrievals for a noise-free $b_{b} / a$ vector, but the introduction of statistical variability very rapidly degraded the matching process. Two sources of uncertainty were considered; (i) the addition of Gaussian noise to the observations and (ii) relaxation of the magnitude of the RMSE error accepted. It was important to determine whether the introduction of a small amount of noise or modest relaxation of the matching criteria led to a catastrophic failure in concentration retrievals or simply to a gradual and proportionate reduction in quality. This is equivalent to the question posed by Defoin-Platel and Chami regarding the shape of the "spectral neighborhood". Figures 3 and 4 show that on average, degradation in OSM retrieval is gradual for small noise additions - in other words, most wrongly identified vectors are associated with OSM triplets which are close to the real values. However, the quality of retrieval degrades very rapidly as noise levels increase. We conclude that the general problem of deriving unambiguous OSM concentrations from SeaWiFs remote sensing spectra in shelf seas which contain a wide range of independently varying OSM concentrations is unlikely to be achievable by spectral matching techniques. It is possible, however, that the number of acceptable solutions to the inversion problem can be reduced $a$ priori by the availability of ancillary information. For example, there may be regions where only a restricted range of OSM concentrations is physically feasible, where particular constituents such as MSS or CDOM dominate the spectral signature, or where a historical time series can be used to constrain solutions. Since marine reflectance spectra are generally devoid of distinctive features (with the exception of the chlorophyll fluorescence peak at around $685 \mathrm{~nm}$ ), our conclusion can probably be extended to other multi-band radiometers such as MODIS (NASA) and MERIS (ESA). For hyperspectral observations, the increased definition of the chlorophyll fluorescence peak may provide an alternative route to OSM concentration recovery.

\section{ACKNOWLEDGEMENTS}

We thank Claire Neil, Ian Brown and David McKee for useful discussions on the ambiguity problem. Danielle Creanor is supported by a UK EPSRC research studentship.

\section{References}

[1] T. Dickey, M. Lewis, and G. Chang, "Optical oceanography: Recent advances and future directions using global remote sensing and in situ observations" Rev. Geophys. 44, RG1001 (2006).

[2] T. Platt, N. Hoepffner, V. Stuart, and C. Brown, Why Ocean Colour? The Societal Benefits of Ocean Colour Technology (Report Number 7, International Ocean Colour Coordinating Group, Dartmouth, Canada, 2008). 
[3] W. Petersen, H. Wehde, H. Krasemann, F. Colijn, and F. Schroeder, "FerryBox and MERIS Assessment of coastal and shelf sea ecosystems by combining in situ and remotely sensed data" Estuar. Coast Shelf S. 77, 296-307 (2008).

[4] I. S. Robinson, Remote sensing of shelf sea ecosystems (Position Paper 12, European Science Foundation, Ostend, Belgium, 2008).

[5] A. Morel, and L. Prieur, "Analysis of variations in ocean colour" Limnol. Oceanogr. 22, 709-722 (1977).

[6] J. T. 0. Kirk, "The relationship between the inherent and the apparent optical properties of surface waters and its dependence on the shape of the volume scattering function" in Ocean Optics, R. W. Spinrad, K. L. Carder, M. J. Perry, eds., (Oxford University Press, 0xford, 1994).

[7] C. D. Mobley, Light and Water: Radiative Transfer in Natural Waters (Academic Press, San Diego, 1994).

[8] C. D. Mobley, "Estimation of the remote-sensing reflectance from above-surface measurements" Appl. 0pt. 38, 7442-7455 (1999).

[9] J. A. Richards, and X. Jia, Remote Sensing Digital Image Analysis: An Introduction (Springer-Verlag, Berlin, 2005).

[10] H. Schiller, and R. Doerffer, "Neural network for emulation of an inverse model - operational derivation of case II water properties from MERIS data" Int. J. Remote Sens. 20, 17351746 (1999).

[11] T. S. Kostadinov, D. A. Siegel, S. Maritorena, and N. Guillocheau, "Ocean color observations and modeling for an optically complex site: Santa Barbara Channel, California, USA" J. Geophys. Res. 112, C07011 (2007).

[12] S. Maritorena, D. A. Siegel, and A. R. Peterson, "Optimization of a semianalytical ocean color model for global-scale applications"
Appl. 0pt. 41, 2705-2714 (2002).

[13] D. A. Siegel, S. Maritorena, N. B. Nelson, and M. J. Behrenfeld, "Independence and interdependencies among global ocean color properties : Reassessing the bio-optical assumption" J. Geophys. Res. 110, C07011 (2005).

[14] C. D. Mobley, L. K. Sundman, C. 0. Davis, T. V. Downes, R. A. Leathers, M. J. Montes, J. H. Bowles, W. P. Bissett, D. D. R. Kohler, R. P. Reid, E. M. Louchard, and A. Gleason, “Interpretation of hyperspectral remote-sensing imagery via spectrum matching and look-up tables" Appl. 0pt. 44, 3576-3592 (2005).

[15] M. P. Lesser, and C. D. Mobley, "Bathymetry, optical properties, and benthic classification of coral reefs using hyperspectral remote sensing imagery" Coral Reefs 26, 819829 (2007).

[16] M. Defoin-Platel, and M. Chami, "How ambiguous is the inverse problem of ocean color in coastal waters?" J. Geophys. Res. 112, C03004 (2007).

[17] M. Babin, D. Stramski, G. M. Ferrari, H. Claustre, A. Bricaud, G. Obolensky, and N. Hoepffner, "Variations in the light absorption coefficients of phytoplankton, nonalgal particles, and dissolved organic matter in coastal waters around Europe" J. Geophys. Res. 108, 3211 (2003).

[18] D. McKee, and A. Cunningham, "Identification and characterisation of two optical water types in the Irish Sea from in situ inherent optical properties and seawater constituents" Estuar. Coast Shelf S. $68,305-316$ (2006).

[19] W. W. Gregg, N. W. Casey, J. E. O'Reilly, and W. E. Esias, “An empirical approach to ocean colour data: reducing bias and the need for post-launch radiometric re-calibration" Remote Sens. Environ. 113, 1598-1612 (2009). 